\title{
Article \\ Effects of a Short-Term "Fat Adaptation with Carbohydrate Restoration" Diet on Metabolic Responses and Exercise Performance in Well-Trained Runners
}

\author{
Kaixuan Che ${ }^{1}$, Junqiang Qiu ${ }^{1, *}$, Longyan Yi ${ }^{1}$, Menghui Zou ${ }^{2}$, Zhihui Li ${ }^{1}$, Amelia Carr ${ }^{3}$, Rhiannon M.J. Snipe ${ }^{3}$ (D) \\ and Dan Benardot 4,5
}

\section{check for} updates

Citation: Che, K.; Qiu, J.; Yi, L.; Zou, M.; Li, Z.; Carr, A.; Snipe, R.M.J.; Benardot, D. Effects of a Short-Term "Fat Adaptation with Carbohydrate Restoration" Diet on Metabolic Responses and Exercise Performance in Well-Trained Runners. Nutrients 2021, 13, 1033. https://doi.org/ $10.3390 /$ nu13031033

Academic Editors: Antoni Sureda and David C. Nieman

Received: 12 February 2021

Accepted: 18 March 2021

Published: 23 March 2021

Publisher's Note: MDPI stays neutral with regard to jurisdictional claims in published maps and institutional affiliations.

Copyright: (c) 2021 by the authors. Licensee MDPI, Basel, Switzerland. This article is an open access article distributed under the terms and conditions of the Creative Commons Attribution (CC BY) license (https:/ / creativecommons.org/licenses/by/ $4.0 /)$.
1 Department of Exercise Biochemistry, Exercise Science School, Beijing Sport University, Beijing 100084, China; 2019210241@bsu.edu.cn (K.C.); 2136@bsu.edu.cn (L.Y.); LiZhiHui0817@hotmail.com (Z.L.)

2 China Athletics School, Beijing Sport University, Beijing 100084, China; zoumh@bsu.edu.cn

3 Centre for Sport Research, Faculty of Health, Deakin University, Melbourne, VIC 3125, Australia; amelia.carr@deakin.edu.au (A.C.); r.snipe@deakin.edu.au (R.M.J.S.)

4 Department of Nutrition, Georgia State University, Atlanta, GA 30303, USA; dan.benardot@emory.edu

5 Center for the Study of Human Health, Emory University, Atlanta, GA 30322, USA

* Correspondence: qiujunqiang@bsu.edu.cn

\begin{abstract}
Periodized carbohydrate availability can enhance exercise capacity, but the effects of shortterm fat adaptation carbohydrate restoration (FACR) diets on metabolic responses and exercise performance in endurance athletes have not been conclusively determined. This study aimed to investigate the effect of a FACR diet on measures of resting metabolism, exercise metabolism, and exercise performance. Well-trained male runners $(n=8)$ completed a FACR dietary intervention (five days' carbohydrate $<20 \%$ and fat $>60 \%$ energy, plus one-day carbohydrate $\geq 70 \%$ energy), and a control high-carbohydrate $(\mathrm{HCHO})$ diet for six days (carbohydrate $>60 \%$ energy; fat $<20 \%$ energy) in a randomized crossover design. Pre- and post-intervention metabolic measures included resting metabolic rate (RMR), respiratory quotient $(R Q)$, maximum fat oxidation rate during exercise (MFO), and maximum fat oxidation intensity (FATmax). Measures of exercise performance included maximal oxygen uptake ( $\left.\mathrm{VO}_{2} \mathrm{max}\right)$, running economy (RE), and $5 \mathrm{~km}$ running time trial $(5 \mathrm{~km}-\mathrm{TT})$. In FACR compared with $\mathrm{HCHO}$, there were significant improvements in FATmax $(p=0.006)$ and RE $(p=0.048)$. There were no significant differences $(p>0.05)$ between FACR and HCHO in RMR, RQ, $\mathrm{VO}_{2} \mathrm{max}$, or $5 \mathrm{~km}$-TT. Findings suggest that a short-term (six days) FACR diet may facilitate increased fat oxidation and submaximal exercise economy but does not improve $5 \mathrm{~km}$-TT performance.
\end{abstract}

Keywords: periodized nutrition; high carbohydrate; high fat diet; glycogen restoration

\section{Introduction}

A periodized carbohydrate intake strategy requires athletes to systematically incorporate periods of low carbohydrate intake (e.g., days or weeks), with high carbohydrate intake implemented at specified time points to support key training sessions [1]. This dietary strategy has in some cases been implemented by athletes [2,3], and may change substrate utilization (e.g., increase fat oxidation) [4], support endurance exercise training adaptations [5], and improve high-intensity exercise performance in sub-elite athletes [1]. One nutritional strategy that can be implemented to achieve a periodized carbohydrate intake is the administration of a non-ketogenic high-fat, low-carbohydrate diet (a restricted carbohydrate availability diet, which also retains sufficient carbohydrate to avoid sustained ketosis [6]) for a specified period of time for adapting to increased fat metabolism, followed by a short period of high carbohydrate intake (carbohydrate restoration; [6]).

Over the past twenty years, short-term fat-adaptation/carbohydrate restoration (FACR) diets have been investigated, and typically encompass at least five days of $15-20 \%$ energy intake from carbohydrate, and 60-65\% energy from fat, followed by $24 \mathrm{~h}$ of a high- 
carbohydrate diet (10-12 g/kg per day; [6]). Reported effects of FACR interventions include an increased contribution of fat as a substrate for exercise performance, via increased rates of whole-body fat oxidation [5], and increases in intra-muscular triglyceride stores, fatty acid mobilization, and transport. Performance in shorter duration endurance running events, such as five km running efforts, after FACR diets have yet to be investigated.

High-fat, low-carbohydrate dietary interventions are typically challenging to implement within both research and practical contexts, due to the substantial changes required to modify typical dietary intakes, and the expense and limited availability of specific foods [7]. Recently, there has been an increased prevalence in the use of smartphone applications, which can assist with dietary prescription and monitoring nutritional intake [8,9]. Such innovations may enable enhanced practitioner dietary prescription and dietary evaluation for athletes to adhere to challenging dietary strategies, such as a FACR dietary intervention, especially if they choose to implement such dietary strategies at specific time points, such as prior to key sporting events or races.

It is currently unclear as to the effects of a six-day FACR dietary intervention on $5 \mathrm{~km}$ running, which is amongst the endurance running events with the highest global participation rates [10], and measures of fat and carbohydrate oxidation. Therefore, this study presents a preliminary investigation of a practical model for the implementation of a FACR dietary intervention, using recently developed technologies (mobile phone applications). The aim of this pilot study was to determine the effect of a short-term FACR diet as prescribed using a mobile phone application on measures of carbohydrate and fat oxidation, and physical performance capacity, in the context of a $5 \mathrm{~km}$ running time trial event.

\section{Materials and Methods}

\subsection{Participants}

Well-trained runners were recruited by advertising to marathon running clubs. A pre-screening questionnaire, which assessed medical, dietary, training, and running competition histories, was completed by well-trained runners to determine eligibility for inclusion in the study. Inclusion criteria comprised healthy male endurance runners aged 18-50 years, regular eating habits without following specific diet types (e.g., ketogenic diet or low carbohydrate diet), and national first-class or second-class athletes (finalists in provincial competitions within China) for running events $>800 \mathrm{~m}$. Eight well-trained runners met the inclusion criteria (Table 1) and included gold medalists in the $5000 \mathrm{~m}$ event (time $15 \mathrm{~min}, 40 \mathrm{~s}$ ) of the 14th Beijing Games in 2014; the gold medalist in the $800 \mathrm{~m}$ event (personal best time $1 \mathrm{~min} 53 \mathrm{~s}$ ) and $1500 \mathrm{~m}$ event (personal best time $4 \mathrm{~min} 4 \mathrm{~s}$ ) in the 2014-2016 Hebei Elite Athletics Championship, and/or top 10\% finish in a sanctioned marathon (e.g., a gold medalist of the 2018 Xuzhou International Half Marathon, with a score of $1 \mathrm{~h}, 8 \mathrm{~min}, 6 \mathrm{~s})$.

Table 1. Participant characteristics, expressed as $\bar{x}$ (mean) and SD (standard deviation); male endurance athletes; $n=8$.

\begin{tabular}{cc}
\hline & $(\bar{x} \pm \mathbf{S D})$ \\
\hline Age (year) & $27 \pm 13$ \\
\hline Height $(\mathrm{cm})$ & $177.1 \pm 5.3$ \\
\hline Body mass $(\mathrm{kg})$ & $65.6 \pm 6.3$ \\
\hline BMI $\left(\mathrm{kg} / \mathrm{m}^{2}\right)$ & $20.9 \pm 1.8$ \\
\hline Body fat $(\%)$ & $13.1 \pm 7.1$ \\
\hline $\mathrm{VO}_{2} \max \left(\mathrm{ml} \cdot \mathrm{kg} \cdot \mathrm{min}^{-1}\right)$ & $47.08 \pm 8.59$ \\
\hline
\end{tabular}

BMI: body mass index; $\mathrm{VO}_{2}$ max: maximal oxygen uptake. 
The research proposal was conducted in compliance with the declaration of Helsinki and approved by the Institutional Review Board of Beijing Sport University (BSU IRB). All participants gave written informed consent prior to study participation (No. 2018025H).

\subsection{Experimental Testing Overview}

Baseline measures, consisting of a three-day food record and body composition assessment (Inbody 720, Biospace Co., Ltd., Seoul, Korea), were completed at baseline prior to experimental testing. Participants then completed two dietary interventions that included an experimental FACR diet and a control high carbohydrate (HCHO) diet in a randomized crossover design with a seven-day washout period (Figure 1). Testing was completed over two days at baseline and following each dietary intervention. Day one of testing included measures of resting metabolic rate $(\mathrm{RMR} ; \mathrm{kcal})$ and respiratory quotient $\left(\mathrm{RQ} ; \mathrm{VCO}_{2} / \mathrm{VO}_{2}\right)$ followed by a six-minute sub-maximum exercise test at a treadmill speed of $11.5 \mathrm{~km} \cdot \mathrm{h}^{-1}$ to determine running economy $\left(\mathrm{RE} ; \mathrm{mL} \cdot \mathrm{kg} \cdot \mathrm{min}^{-1}\right)$, which is quantified as the $\mathrm{O}_{2}$ cost for a given velocity, estimated from measuring steady-state $\mathrm{O}_{2}$ consumption $\left(\mathrm{VO}_{2}\right)$ during submaximal running [11]. It has been established that runners with a higher RE will record a lower $\mathrm{VO}_{2}$ at a given steady-state speed than runners with a lower RE [12]. In addition, a maximal exercise test was performed to determine maximal oxygen consumption $\left(\mathrm{VO}_{2} \mathrm{max} ; \mathrm{mL} \cdot \mathrm{kg} \cdot \mathrm{min}^{-1}\right)$ and maximum fat oxidation $\left(\mathrm{MFO} ; \mathrm{g} \cdot \mathrm{min}^{-1}\right)$. On day two of testing, participants completed a $5 \mathrm{~km}$ running time trial $(5 \mathrm{~km}-\mathrm{TT})$ test (Figure 1$)$.

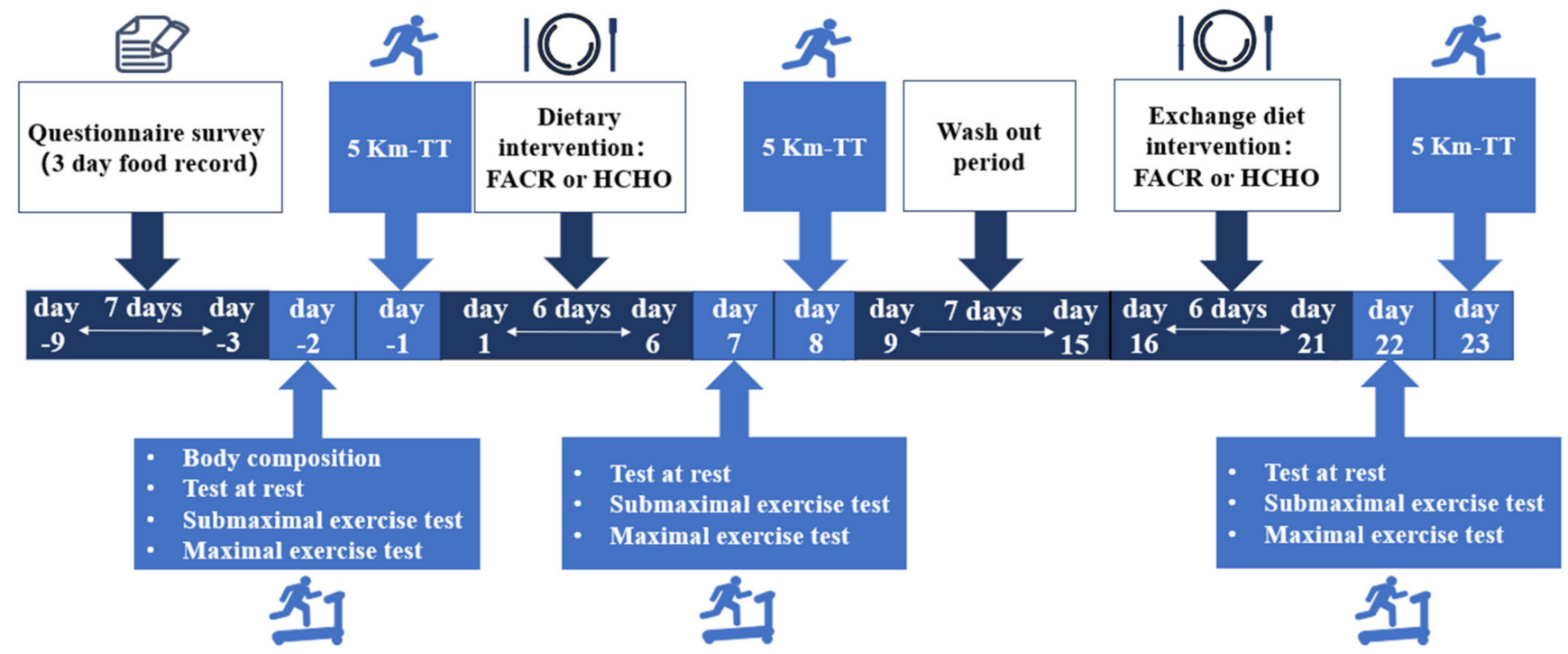

Figure 1. Experimental testing overview. The test at rest and exercise test were performed three times, that is, before any intervention, then after fat adaptation carbohydrate restoration (FACR) (or control high-carbohydrate (HCHO)), and then after HCHO (or FACR). Resting metabolic rate (RMR) and respiratory quotient (RQ) were measured in the test at rest. Maximum fat oxidation rate (MFO), maximum fat oxidation intensity (FATmax), oxygen consumption $\left(\mathrm{VO}_{2}\right)$ under FATmax $\left(\mathrm{VO}_{2}\right.$ (FATmax)), running speed under FATmax (Speed (FATmax)), running economy (RE), heart rate $(\mathrm{HR}), \mathrm{VO}_{2} \mathrm{max}$ and $5 \mathrm{~km}$ time trial (5-km TT) were measured in exercise tests.

\subsection{Experimental Protocol}

\subsubsection{Diet Plan}

Participants completed a three-day food record of their habitual diet over two weekdays and one weekend day [13] to determine baseline nutrient (\% energy intake from carbohydrate, fat, and protein) intake one week prior to the experimental intervention. Participants were familiarized with the nutrient analysis application (Boohee, Information Technology Co., Ltd., Shanghai, China) $[14,15]$ that was installed on the participant's smartphone and used to complete the three-day food record. Portion sizes for all consumed 
foods and liquids were estimated using standard-sized bowls and dishes as the unit, with the nutrient analysis provided by the application.

The application was used to develop personalized recipe suggestions for each participant, based on their habitual intake and prescribed dietary interventions, which consisted of (1) a five-day non-ketogenic, high-fat ( $>60 \%$ energy intake) low-carbohydrate $(<20 \%$ energy intake) diet followed by a one-day high-carbohydrate ( $\geq 70 \%$ energy intake) diet to restore muscle glycogen (FACR) [6]; and (2) a high-carbohydrate (carbohydrate $>60 \%$ energy intake; fat $<20 \%$ energy intake) diet for six days ( $\mathrm{HCHO}$ ) in a randomized crossover design with a seven-day washout period $[16,17]$. Participant adherence to their dietary plan was monitored using the application's diet tracking function that validated intake from photos of food and fluids that were taken and uploaded to the application by each participant. Upon completion of the study, energy intake distributions (\%) from carbohydrate, protein, and fat were downloaded from the application and recorded (Table 2).

Table 2. Carbohydrate, fat and protein intake (\% energy intake), for fat adaptation/carbohydrate restoration (FACR) and high carbohydrate $(\mathrm{HCHO})$ dietary records $(\bar{x} \pm \mathrm{SD})$.

\begin{tabular}{|c|c|c|c|c|}
\hline & \multirow{2}{*}{$\begin{array}{c}\begin{array}{c}\text { Pre- } \\
\text { Intervention }\end{array} \\
1-3 \mathrm{~d}\end{array}$} & \multicolumn{2}{|c|}{ Post-Intervention (FACR) } & \multirow{2}{*}{$\begin{array}{c}\text { Post- } \\
\begin{array}{c}\text { Intervention } \\
(\mathrm{HCHO})\end{array} \\
1-6 \mathrm{~d} \\
\end{array}$} \\
\hline & & $1-5 \mathrm{~d}$ & 6th d & \\
\hline $\mathrm{CHO}(\%)$ & $52.3 \pm 5.8$ & $23.4 \pm 4.9 * * \# \#$ & $66.3 \pm 3.8^{* * \# \#}$ & $59.4 \pm 1.8^{* *}$ \\
\hline FAT $(\%)$ & $31.5 \pm 4.6$ & $58.8 \pm 4.2 * * \# \#$ & $19.1 \pm 4.4^{* * \# \#}$ & $25.2 \pm 2.1 * *$ \\
\hline PRO (\%) & $16.2 \pm 2.0$ & $17.9 \pm 1.7$ *\#\# & $14.6 \pm 1.9^{\# \#}$ & $15.4 \pm 2.3^{* *}$ \\
\hline
\end{tabular}

\subsubsection{Resting Metabolism Test}

During the data collection period, participants performed their normal training at least twice per week, and refrained from any competition for a minimum of seven days prior to testing and were required to rest for $12 \mathrm{~h}$ prior to testing. Participants were required to abstain from alcohol or caffeine for $48 \mathrm{~h}$ prior to each two-day testing period to avoid any potential effects on exercise performance or heart rate. Participants arrived at the laboratory after a 10-h overnight fasting period and sat in a seated posture for 20 min upon arrival. Resting energy expenditure was quantified via indirect calorimetry (COSMED Quark PFT Ergo, Roma, Italy). Resting metabolic rate (RMR, $\left.\mathrm{kcal} \cdot \mathrm{d}^{-1}\right)$ testing was then conducted, using a metabolic cart (COSMED Quark PFT Ergo, Roma, Italy). The RQ $\left(\mathrm{VCO}_{2} / \mathrm{VO}_{2}\right)$ was recorded and RMR $\left(\mathrm{kcal} \cdot \mathrm{d}^{-1}\right)$ was calculated by the Weir formula [18].

\subsubsection{Submaximal Exercise Protocol}

Upon completion of the resting metabolism test, participants performed a submaximal running test on a motorized treadmill ( $\mathrm{h} / \mathrm{p} /$ cosmos pulsar, Munich, Germany) to determine running economy (RE). The test consisted of a three-minute warm-up, at a speed of five $\mathrm{km} \cdot \mathrm{h}^{-1}$ and a gradient of zero followed by six minutes running at a treadmill speed of $11.5 \mathrm{~km} \cdot \mathrm{h}^{-1}$. Oxygen consumption $\left(\mathrm{mL} \cdot \mathrm{kg} \cdot \mathrm{min}^{-1}\right)$ was collected for a three-minute period once steady-state $\mathrm{VO}_{2}$ was reached, and this value was recorded as the RE [19]. Heart rate $(\mathrm{HR}, \mathrm{bpm})$ was measured continuously during this period and the mean value during the three-minute steady state was recorded (Polar V800, Polar, NY, USA).

\subsubsection{Maximal Aerobic Capacity}

Maximal oxygen uptake $\left(\mathrm{VO}_{2} \mathrm{max}\right)$ testing was conducted on a motorized treadmill following completion of the submaximal running test, and a 30-min rest period. The protocol began with a three-minute walking warm-up at $5 \mathrm{~km} \cdot \mathrm{h}^{-1}$. The starting speed for the test was $6.8 \mathrm{~km} \cdot \mathrm{h}^{-1}$, followed by three-minute incremental stages with a $1.2 \mathrm{~km} \cdot \mathrm{h}^{-1}$ 
increase in speed at each stage, and the gradient remained unchanged at zero throughout the process. The protocol used was similar to previously reported protocols $[20,21]$. The criteria for attaining $\mathrm{VO}_{2}$ max was achieving at least three of the following criteria: (1) $\mathrm{VO}_{2}$ did not increase with the increase of load, and the participants voluntarily stopped the treadmill when they were exhausted; (2) the variation range of $\mathrm{VO}_{2}$ did not exceed $5 \%$ or $150 \mathrm{~mL} \cdot \mathrm{min}^{-1}$ or $2 \mathrm{~mL} \cdot \mathrm{kg} \cdot \mathrm{min}^{-1}$; (3) RQ > 1.1; and (4) HR > $180 \mathrm{bpm}$.

During the test, ventilation samples were collected continuously using an indirect calorimetry system, (COSMED Quark PFT Ergo, Roma, Italy), which was calibrated prior to each test. $\mathrm{VO}_{2}$ and $\mathrm{VCO}_{2}$ were recorded every $30 \mathrm{~s}$ and used to calculate fat oxidation rates [22]. Maximum fat oxidation rate (MFO, g. $\mathrm{min}^{-1}$ ) per unit fat mass was determined as the highest recorded value of the last $30 \mathrm{~s}$ interval of each stage, and the corresponding intensity was the maximum fat oxidation exercise intensity (FATmax, $\% \mathrm{VO}_{2} \mathrm{max}$ ). MFO during exercise has been widely reported and can reflect the ability to maximize fat utilization [23]. In addition, $\mathrm{VO}_{2}\left(\mathrm{~mL} \cdot \mathrm{kg} \cdot \mathrm{min}^{-1}\right)$ and running speed $\left(\mathrm{km} \cdot \mathrm{h}^{-1}\right)$ at the same intensity were recorded. Heart rate (Polar V800, Polar, NY, USA) was measured throughout the test.

\subsubsection{Five km Time Trial}

Participants followed the diet consistent with their last day of treatment (FACR or $\mathrm{HCHO}$ ) within $24 \mathrm{~h}$ before the $5 \mathrm{~km}$-TT (e.g., high in carbohydrate under both conditions) to restore muscle glycogen and included a high-carbohydrate breakfast. On day two of testing, participants returned to the laboratory. After a 20-min rest period, participants completed a five-minute warm-up at a self-selected speed. Participants then performed an individual $5 \mathrm{~km}$ running time trial on an outdoor synthetic track-and-field ground, and the time was recorded using a stopwatch (CASIO HS-70W, Tokyo, Japan).

\subsection{Statistical Analyses}

Statistical analyses were undertaken using SPSS 17.0. For baseline data (Table S1), a paired samples $t$-tests were used to compare test data (RMR, RQ, MFO, FATmax, $\mathrm{VO}_{2}$ (FATmax), Speed (FATmax), RE, HR(RE), $\mathrm{VO}_{2} \max , 5 \mathrm{~km}-\mathrm{TT}$ ) between baseline and the dietary interventions (FACR or HCHO). Data from the two dietary conditions (Tables S2 and S3) were compared using a two-factor (diet and time) analysis of variance (ANOVA) with repeated measures. Tukey-Kramer post hoc tests were undertaken when ANOVA revealed a significant difference or interaction effects were observed. Significance was accepted at $p<0.05$. All data are reported as $\bar{x} \pm \mathrm{SD}$ (Table S4).

\section{Results}

\subsection{MFO and FATmax}

There was a significant increase in MFO from pre to post-intervention in FACR $\left(0.36 \pm 0.12 \mathrm{~g} \cdot \mathrm{min}^{-1} \mathrm{vs} .0 .46 \pm 0.06 \mathrm{~g} \cdot \mathrm{min}^{-1}, p=0.037\right)$, but no significant difference was observed in $\mathrm{HCHO}\left(0.36 \pm 0.12 \mathrm{~g} \cdot \mathrm{min}^{-1}\right.$ vs. $\left.0.45 \pm 0.19 \mathrm{~g} \cdot \mathrm{min}^{-1}, p=0.258\right)$ or between FACR and $\mathrm{HCHO}\left(0.46 \pm 0.06 \mathrm{~g} \cdot \mathrm{min}^{-1}\right.$ vs. $\left.0.45 \pm 0.19 \mathrm{~g} \cdot \mathrm{min}^{-1}, p=0.919\right)$ (Figure 2a). There was no significant increase in FATmax from pre to post-intervention in FACR $(61.27 \pm 9.26 \%$ $\mathrm{VO}_{2} \max$ vs. $\left.64.74 \pm 8.87 \% \mathrm{VO}_{2} \max , p=0.341\right)$ and $\mathrm{HCHO}\left(61.27 \pm 9.26 \% \mathrm{VO}_{2}\right.$ max vs. $\left.57.79 \pm 7.57 \% \mathrm{VO}_{2} \max , p=0.171\right)$. However, FATmax was significantly higher for FACR compared with $\mathrm{HCHO}\left(64.74 \pm 8.87 \% \mathrm{VO}_{2} \max\right.$ vs. $\left.57.79 \pm 7.57 \% \mathrm{VO}_{2} \max , p=0.006\right)$ (Figure 2b).

\subsection{Resting Metabolism}

There was no significant difference in RMR from pre to post-intervention in FACR $\left(1968.19 \pm 383.68 \mathrm{kcal} \cdot \mathrm{d}^{-1} \mathrm{vs} .2051 .21 \pm 375.68 \mathrm{kcal} \cdot \mathrm{d}^{-1}, p=0.625\right)$ and HCHO $\left(1968.19 \pm 383.68 \mathrm{kcal} \cdot \mathrm{d}^{-1}\right.$ vs. $\left.2017.81 \pm 397.00 \mathrm{kcal} \cdot \mathrm{d}^{-1}, p=0.797\right)$ or between FACR and HCHO $\left(2051.21 \pm 375.68 \mathrm{kcal} \cdot \mathrm{d}^{-1}\right.$ vs. $\left.2017.81 \pm 397.00 \mathrm{kcal} \cdot \mathrm{d}^{-1}, p=0.707\right)$ (Figure 3a). There was no significant difference in RQ from pre to post-intervention in 
FACR $(0.84 \pm 0.07$ vs. $0.82 \pm 0.05, p=0.430)$ and $\mathrm{HCHO}(0.84 \pm 0.07$ vs. $0.83 \pm 0.06$, $p=0.613$ ) (Figure $3 b)$ or between FACR and HCHO (0.82 \pm 0.05 vs. $0.83 \pm 0.06$, $p=0.647)$.

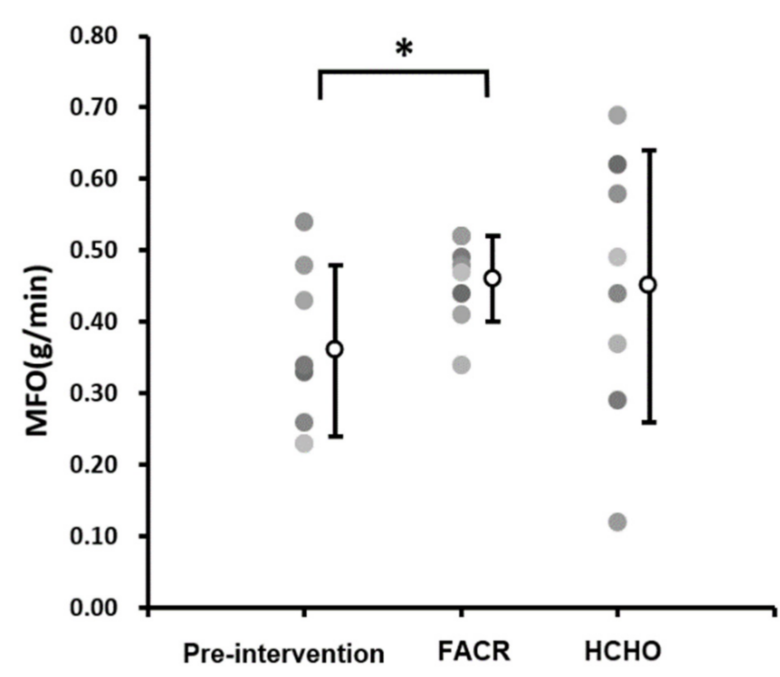

(a)

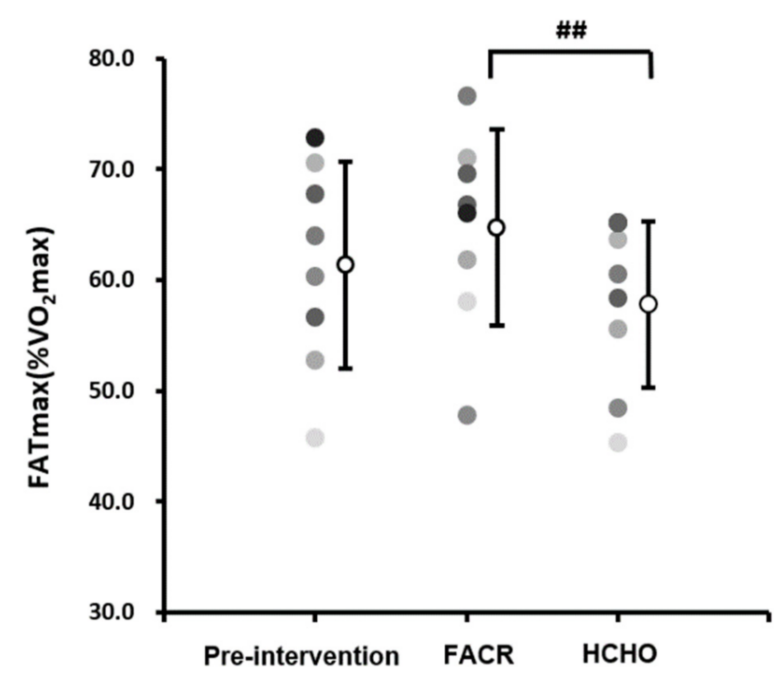

(b)

Figure 2. Individual (a) maximum fat oxidation rate (MFO) and (b) maximum fat oxidation intensity (FATmax) during a maximal graded treadmill test pre-intervention and after fat adaptation/carbohydrate restoration (FACR) and high carbohydrate $(\mathrm{HCHO})$ diets. * Significantly different to pre-intervention $(p<0.05)$. \#\# Significantly different to HCHO control $(p<0.01)$.

\section{3. $\mathrm{VO}_{2}$ (FATmax) and Speed (FATmax)}

There was no significant difference in $\mathrm{VO}_{2}$ (FATmax) from pre to post-intervention in FACR $\left(28.73 \pm 6.05 \mathrm{~mL} \cdot \mathrm{kg} \cdot \mathrm{min}^{-1}\right.$ vs. $\left.29.94 \pm 4.98 \mathrm{~mL} \cdot \mathrm{kg} \cdot \mathrm{min}^{-1}, p=0.197\right)$ and HCHO $\left(28.73 \pm 6.05 \mathrm{~mL} \cdot \mathrm{kg} \cdot \mathrm{min}^{-1}\right.$ vs. $\left.28.55 \pm 7.23 \mathrm{~mL} \cdot \mathrm{kg} \cdot \mathrm{min}^{-1}, p=0.220\right)$ or between FACR and HCHO $\left(29.94 \pm 4.98 \mathrm{~mL} \cdot \mathrm{kg} \cdot \mathrm{min}^{-1}\right.$ vs. $\left.28.55 \pm 7.23 \mathrm{~mL} \cdot \mathrm{kg} \cdot \mathrm{min}^{-1}, p=0.479\right)$ (Figure 3c). There was no significant difference in speed (FATmax) from pre to post-intervention change in FACR $(8.60 \pm 2.03 \mathrm{~km} / \mathrm{h}$ vs. $9.50 \pm 2.92 \mathrm{~km} / \mathrm{h}, p=0.285)$ and HCHO $(8.60 \pm 2.03 \mathrm{~km} / \mathrm{h}$ vs. $8.60 \pm 1.92 \mathrm{~km} / \mathrm{h}, p=1.000)$ or between FACR and HCHO $(9.50 \pm 2.92 \mathrm{~km} / \mathrm{h}$ vs. $8.60 \pm 1.92 \mathrm{~km} / \mathrm{h}, p=0.176$ ) (Figure $3 \mathrm{~d}$ ).

\section{4. $R E, H R(R E), V O_{2}$ max and Five $k m-T T$}

There was no significant difference in RE (running economy) from pre to postintervention in FACR $\left(36.07 \pm 2.65 \mathrm{~mL} \cdot \mathrm{kg} \cdot \mathrm{min}^{-1} \mathrm{vs} .34 .64 \pm 2.54 \mathrm{~mL} \cdot \mathrm{kg} \cdot \mathrm{min}^{-1}, p=0.097\right)$ and $\mathrm{HCHO}\left(36.07 \pm 2.65 \mathrm{~mL} / \mathrm{kg} \cdot \mathrm{min}^{-1}\right.$ vs. $\left.36.11 \pm 3.26 \mathrm{mG} \cdot \mathrm{kg} \cdot \mathrm{min}^{-1}, p=0.967\right)$. However, RE $\left(\mathrm{mL} \cdot \mathrm{kg} \cdot \mathrm{min}^{-1}\right)$ was significantly lower in FACR compared with HCHO $\left(34.64 \pm 2.54 \mathrm{~mL} \cdot \mathrm{kg} \cdot \mathrm{min}^{-1}\right.$ vs. $\left.36.11 \pm 3.26 \mathrm{~mL} \cdot \mathrm{kg} \cdot \mathrm{min}^{-1}, p=0.048\right)$ (Figure 3e). There was a significant decrease in HR(RE) from pre to post-intervention in FACR $(142.13 \pm 16.75 \mathrm{bpm}$ vs. $134.38 \pm 11.01 \mathrm{bpm}, p=0.048)$; but not in $\mathrm{HCHO}(142.13 \pm 16.75 \mathrm{bpm}$ vs. $138.25 \pm 13.54 \mathrm{bpm}$, $p=0.566)$. HR(RE) in FACR was not significantly different to $\mathrm{HCHO}(134.38 \pm 11.01 \mathrm{bpm}$ vs. $138.25 \pm 13.54 \mathrm{bpm}, p=0.440$ ) (Figure 3f).

There was no significant difference in $\mathrm{VO}_{2}$ max from pre to post-intervention in FACR $\left(47.08 \pm 8.59 \mathrm{~mL} \cdot \mathrm{kg} \cdot \mathrm{min}^{-1}\right.$ vs. $\left.48.12 \pm 7.30 \mathrm{~mL} \cdot \mathrm{kg} \cdot \mathrm{min}^{-1}, p=0.491\right)$ and HCHO $\left(47.08 \pm 8.59 \mathrm{~mL} \cdot \mathrm{kg} \cdot \mathrm{min}^{-1}\right.$ vs. $\left.47.36 \pm 5.78 \mathrm{~mL} \cdot \mathrm{kg} \cdot \mathrm{min}^{-1}, p=0.847\right)$ or between FACR and $\mathrm{HCHO}\left(48.12 \pm 7.30 \mathrm{~mL} \cdot \mathrm{kg} \cdot \mathrm{min}^{-1}\right.$ vs. $\left.47.36 \pm 5.78 \mathrm{~mL} \cdot \mathrm{kg} \cdot \mathrm{min}^{-1}, p=0.428\right)$ (Figure $3 \mathrm{~g}$ ). There was no significant difference in $5 \mathrm{~km}$-TT from pre to post-intervention in FACR $(1210.38 \pm 281.98 \mathrm{~s}$ vs. $1241.38 \pm 306.45 \mathrm{~s}, p=0.116)$ and HCHO $(1210.38 \pm 281.98 \mathrm{~s}$ vs. $1223.00 \pm 307.88 \mathrm{~s}, p=0.662)$ or between FACR and HCHO $(1241.38 \pm 306.45 \mathrm{~s}$ vs. $1223.00 \pm 307.88 \mathrm{~s}, p=0.318$ ) (Figure 3h). 


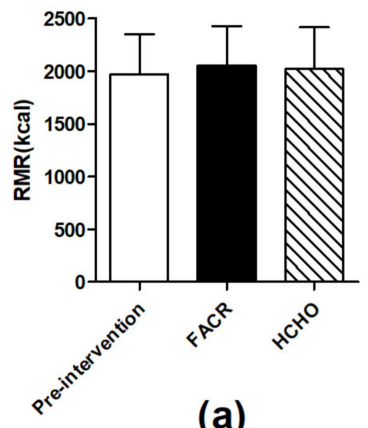

(a)

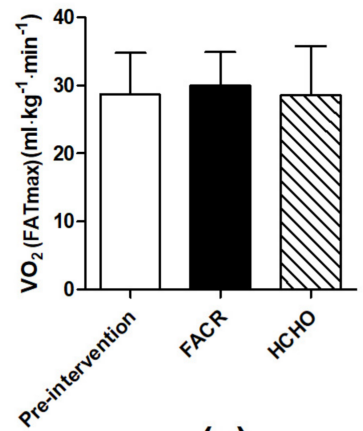

(c)

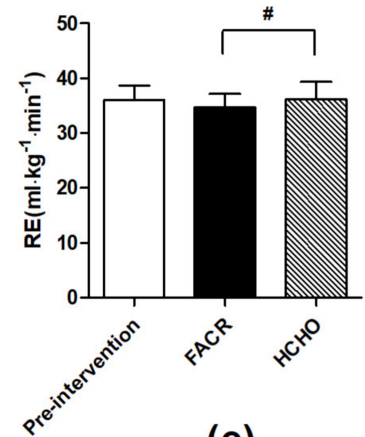

(e)

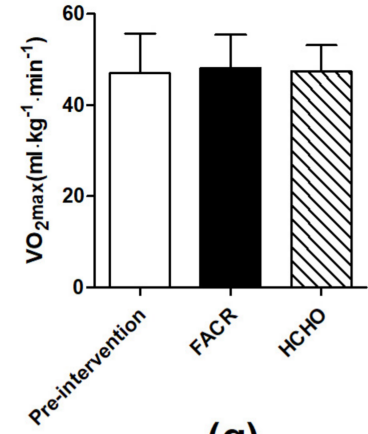

(g)

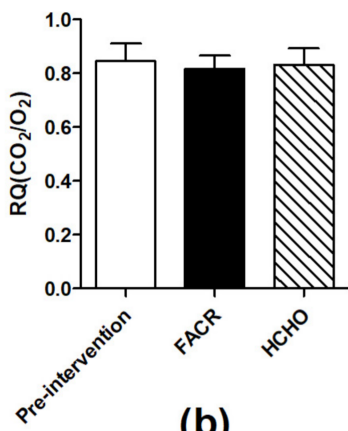

(b)

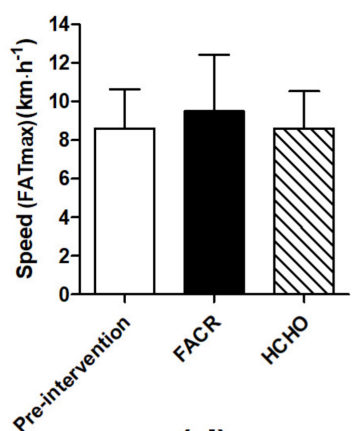

(d)

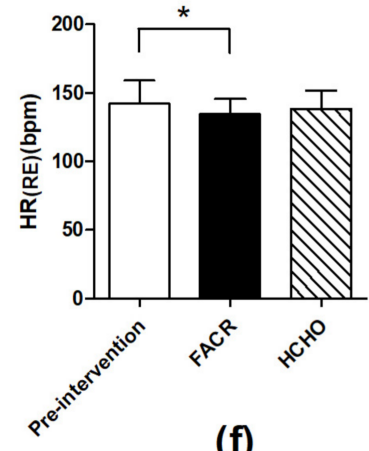

(f)

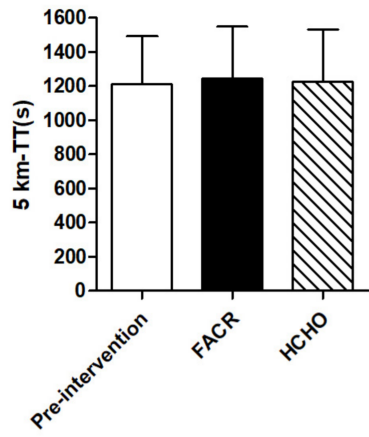

(h)

Figure 3. (a) Resting metabolic rate (RMR), (b) respiratory quotient (RQ), (c) $\mathrm{VO}_{2}$ under $F A T \max \left(\mathrm{VO}_{2}\right.$ (FATmax)), (d) running speed under FATmax (Speed (FATmax)), (e)running economy (RE), (f) heart rate under $\mathrm{RE}(\mathrm{HR}(\mathrm{RE}))$, (g) $\mathrm{VO}_{2}$ max and (h) $5 \mathrm{~km}$ time trial (5-km TT) pre-intervention and after fat adaptation/carbohydrate restoration (FACR) and high carbohydrate (HCHO) diets. * Significantly different to pre-intervention $(p<0.05)$. \# Significantly different to HCHO control $(p<0.05)$. 


\section{Discussion}

In this pilot study, we investigated a practical model for implementing a short-term fat adaptation followed by carbohydrate restoration (FACR) diet in well-trained runners. Consistent with previous studies in the area, we also investigated markers of carbohydrate and fat metabolism and exercise performance. Key findings were a significant increase in the exercise intensity at which maximal fat oxidation rates occurred (FATmax), a significant increase in running economy, in FACR compared with a high carbohydrate (HCHO) control, and a significant increase in maximal fat oxidation rates from pre to post intervention in the FACR condition. No significant changes were detected in markers of carbohydrate metabolism, or $5 \mathrm{~km}$ time trial performance.

In the current investigation, maximal fat oxidation rates were achieved at a higher exercise intensity in FACR than with $\mathrm{HCHO}$ control, evidenced by a significantly higher FATmax after FACR. Given that under normal dietary conditions there is typically a decrease in fat oxidation rates as exercise intensity increases, the results of the current pilot study provide a preliminary indication that there may be differences in substrate selection $[20,24]$ following the FACR intervention. Further studies on the effects of a FACR intervention on FATmax are required, however, given the small sample size used in the current study. Previous studies investigating FACR have not quantified FATmax but have investigated fat oxidation rates. In previous studies with similar, non-ketogenic FACR protocols to the current study, increased rates of fat oxidation and reduced carbohydrate oxidation rates have been observed during submaximal cycling $[4,25,26]$.

In the current investigation, no significant differences were observed between FACR and control in maximal fat oxidation rates. The use of noninvasive methods of quantifying fat oxidation in the current pilot investigation may provide direction for future studies, which may use larger sample sizes and more direct methods. However, there was a significant difference in maximal fat oxidation rates in the FACR condition, between the pre-and post-intervention time points. The trend toward increased fat metabolism and reduced carbohydrate oxidation in endurance athletes after FACR, as indicated by the preliminary results provided by the current pilot study, and in previous studies $[4,26-29]$ is suggestive of diet-induced adaptations that could enhance the training-induced increased capacity for fat adaptation observed in endurance-trained athletes [5].

Running economy in the current investigation was significantly improved in the FACR compared with the $\mathrm{HCHO}$ condition. This is the first study to investigate the effects of a FACR intervention on running economy, however, several previous studies have investigated similar measures of exercise economy and efficiency after high-fat dietary interventions $[19,29,30]$. In contrast to the findings of the current investigation, a recent study investigating elite race walkers after a short-term ketogenic diet (defined as severe carbohydrate restriction, and induced chronic ketosis [6]), and carbohydrate restoration period reported impaired exercise economy [29]. Similarly, submaximal running efficiency was impaired after a 31-day ketogenic diet intervention in trained endurance athletes [31]. In both previous studies, the ketogenic diet intervention (in contrast with the non-ketogenic high fat diet used in the current investigation) and increased reliance on fat oxidation may partially explain the increased oxygen cost when exercising at specific exercise intensities, given the substantially reduced carbohydrate availability associated with the substantially restricted carbohydrate intake typical of ketogenic diets $(<50 \mathrm{~g} /$ day; [6]). The results presented within this study suggest that a non-ketogenic, short-term FACR diet may elicit a beneficial effect on running economy. While running economy is a valid predictor of running performance in runners [19], further investigation is required to address the limitation of small sample size within the current pilot study. It is also important to note that the current investigation found that running performance was maintained rather than improved in comparison to $\mathrm{HCHO}$.

A unique feature of the current investigation was the quantification of performance in a $5 \mathrm{~km}$ time trial, in a setting that replicated real-life racing conditions. There were no significant differences between the FACR and HCHO conditions, and, therefore, no 
detrimental effect on performance after the FACR intervention. No previous study has quantified running performance after FACR, but previous investigations have reported impaired high-intensity exercise capacity, including a reduced power output in a $1 \mathrm{~km}$ cycling time trial after FACR compared with control [32]. The potential for impaired performances in high intensity exercise after FACR may be partially attributable to blunted pyruvate dehydrogenase activation and reduced carbohydrate oxidation [26], given that carbohydrate is the predominant fuel required for high-intensity exercise [30]. Further investigation of the effects on $5 \mathrm{~km}$ running performance is warranted, given that there was some variation in training status within our participant population, as indicted by the standard deviation in $\mathrm{VO}_{2}$ max values.

Other observed responses to high fat diet interventions, such as reduced body mass and improved body composition [33], are also associated with improvements in high intensity running performance [34], which could also contribute to longer term performance outcomes for $5 \mathrm{~km}$ runners. Further investigation of the effect of repeated exposures to FACR over extended periods as a strategy to achieve low carbohydrate availability is warranted, given the prevalence of low carbohydrate availability dietary strategies in athletes [2,3] and reported performance benefits after systematically implemented low carbohydrate availability interventions [1].

The FACR protocol used in the current investigation may have practical benefits for trained runners. A six-day FACR intervention (five-day high fat diet) is likely to be more realistic and feasible to implement than longer term high fat dietary interventions [25]. Further, the use of mobile phone applications, as used in the current investigation, may further assist with the implementation of FACR. Given that a high fat intake requires substantial changes to typical dietary patterns, which for most athletes typically include a high proportion of carbohydrates [35], dietary monitoring is likely to be required, and the increased prevalence of mobile phone applications for dietary standardization [36] may reduce the burden on athletes when documenting their intake [37]. Applications are also typically easier to include within daily training routines than more traditional methods of recording dietary intake [36].

There are some limitations within the current investigation, which provide direction for future research in this area. Future studies should aim to include more direct measures of fat oxidation rates, and the quantification of additional, minimally invasive physiological measures (e.g., blood lactate concentration) using a similar study design would provide further insight into carbohydrate metabolism during exercise (e.g., maximal $5 \mathrm{~km}$ efforts). Future studies investigating FACR and running performance are required to build upon the findings of the current pilot study. Significantly lower mean plasma lactate concentration was previously observed during high intensity cycling training after FACR compared with control [32], and similar measures during a running-based study would facilitate comparisons between exercise modalities after FACR. In addition, no measures of ketones (acetoacetate, beta-hydroxybutyrate, and/or acetone) were undertaken on either the FACR or $\mathrm{HCHO}$ groups to determine dietary influence on ketone production. Rating of perceived exertion (RPE) is another noninvasive method that would provide additional detail to athletes' responses to FACR interventions and high-intensity running, given that cyclingbased studies have reported both consistent RPE for FACR and control conditions, coupled with impaired high-intensity performance, and increased RPE after FACR compared to $\mathrm{HCHO}$ at a fixed exercise intensity [32,38]. Additional subjective measures, which would more comprehensively document runners' responses to FACR in competitive context, include the quantification of side effects, given that an increased severity and/or prevalence of gastrointestinal symptoms is a well-documented response to changes in carbohydrate intake $[39,40]$, and fatigue-related symptoms, which have been reported after FACR in cyclists [25]. The standardization and quantification of training performed prior to and during the FACR intervention would allow further interpretation of the effectiveness of FACR interventions, particularly in the context of training responses quantified after several days of low carbohydrate intake. In the current investigation, there were significant 
differences in macronutrient intakes between the two dietary conditions, however, the mean carbohydrate intake during FACR was slightly higher than the recommended intake for FACR ( $\leq 20 \%$ of total energy intake; [ 6 ] and fat intake was slightly lower than recommended $(>60 \%$; [6]), which is a limitation of the current investigation. A unique element of the current investigation was the use of mobile phone applications to implement the dietary intervention. Potentially, this aspect of the methodology may have impacted participants' ability to reduce their carbohydrate intake and increase fat intake. In future investigations, the use of smartphone applications could be supported by additional education provided to runners by accredited sports dietitians, given that applications are currently used by some dietitians, and the support provided when using applications can improve nutrition knowledge in athletes [41].

\section{Conclusions}

The results of this pilot study indicate that a short-term, non-ketogenic, high fat dietary intervention, followed by $24 \mathrm{~h}$ of carbohydrate restoration (FACR) can enhance measures of fat oxidation and running economy. Running performance after FACR did not differ from that observed in the control condition. However, further investigation is required to explore if $5 \mathrm{~km}$ running performance can be improved with repeated exposures to FACR over extended periods of time.

Supplementary Materials: The following are available online at https:/ / www.mdpi.com/2072-664 3/13/3/1033/s1, Table S1: Individual RMR: RQ, MFO, FATmax, $\mathrm{VO}_{2}$ (FATmax), Speed (FATmax), $\mathrm{RE}, \mathrm{HR}(\mathrm{RE}), \mathrm{VO}_{2} \mathrm{max}$ and 5-km TT of eight participants for pre-intervention. Table S2: Individual RMR, RQ, MFO, FATmax, $\mathrm{VO}_{2}$ (FATmax), Speed (FATmax), RE, HR(RE), $\mathrm{VO}_{2}$ max and 5-km TT of eight participants for FACR. Table S3: Individual RMR, RQ, MFO, FATmax, $\mathrm{VO}_{2}$ (FATmax), Speed (FATmax), RE, $\mathrm{HR}(\mathrm{RE}), \mathrm{VO}_{2}$ max and 5-km TT of eight participants for HCHO. Table S4: Comparing results of RMR, RQ, MFO, FATmax, $\mathrm{VO}_{2}$ (FATmax), Speed (FATmax), RE, HR(RE), $\mathrm{VO}_{2}$ max and 5-km TT for pre and post-intervention (FACR or HCHO).

Author Contributions: K.C. was responsible for the operation of the experiment, data collection, data interpretation, writing and revision of the manuscript, under the direction and assistance of J.Q. who assisted with each step and completion of the manuscript. L.Y. and Z.L. assisted in the completion of the experiment. M.Z. assisted in recruiting participants and participating enthusiastically in this project. D.B., R.M.J.S. and A.C. assisted in the revision of the manuscript. All authors have read and agreed to the published version of the manuscript.

Funding: Funding was provided by the National Key R\&D Program of China (2018YFC2000600).

Institutional Review Board Statement: The research proposal was approved by the Institutional Review Board of Beijing Sport University (BSU IRB) and all participants gave written informed consent prior to study participation (No. 2018025H).

Informed Consent Statement: Informed consent was obtained from all subjects involved in the study.

Data Availability Statement: The following are available online at http:/ / file.for.work/2021-1-31. pdf (accessed on 31 January 2021).

Acknowledgments: The authors would like to sincerely thank all the participants for volunteering their time and efforts during the research investigation. The authors declare no conflict of interest.

Conflicts of Interest: The authors declare no conflict of interest.

\section{References}

1. Marquet, L.-A.; Hausswirth, C.; Molle, O.; Hawley, J.A.; Burke, L.M.; Tiollier, E.; Brisswalter, J. Periodization of carbohydrate intake: Short-term effect on performance. Nutrients 2016, 8, 755. [CrossRef] [PubMed]

2. Heikura, I.A.; Stellingwerff, T.; Mero, A.A.; Uusitalo, A.L.T.; Burke, L.M. A mismatch between athlete practice and current sports nutrition guidelines among elite female and male middle-and long-distance athletes. Int. J. Sport Nutr. Exerc. Metab. 2017, 27, 351-360. [CrossRef] [PubMed]

3. Stellingwerff, T. Case study: Nutrition and training periodization in three elite marathon runners. Int. J. Sport Nutr. Exerc. Metab. 2012, 22, 392-400. [CrossRef] [PubMed] 
4. Yeo, W.K.; Lessard, S.J.; Chen, Z.-P.; Garnham, A.P.; Burke, L.M.; Rivas, D.A.; Kemp, B.E.; Hawley, J.A. Fat adaptation followed by carbohydrate restoration increases AMPK activity in skeletal muscle from trained humans. J. Appl. Physiol. 2008, 105, 1519-1526. [CrossRef]

5. Hawley, J.A.; Leckey, J.; Parr, E.B.; Hoffman, N.J.; Devlin, B.L.; Burke, L.; Trewin, A.J.; Stepto, N.K.; Morton, J.P. High dietary fat intake increases fat oxidation and reduces skeletal muscle mitochondrial respiration in trained humans. FASEB J. 2018, 32, 2979. [CrossRef]

6. Burke, L.M.; Hawley, J.A.; Jeukendrup, A.; Morton, J.P.; Stellingwerff, T.; Maughan, R.J. Toward a common understanding of diet-exercise strategies to manipulate fuel availability for training and competition preparation in endurance sport. Int. J. Sport Nutr. Exerc. Metab. 2018, 28, 451-463. [CrossRef]

7. Mirtschin, J.G.; Forbes, S.F.; Cato, L.E.; Heikura, I.A.; Strobel, N.; Hall, R.; Burke, L.M. Organization of dietary control for nutrition-training intervention involving periodized carbohydrate availability and ketogenic low-carbohydrate high-fat diet. Int. J. Sport Nutr. Exerc. Metab. 2018, 28, 480-489. [CrossRef]

8. Jospe, M.R.; Fairbairn, K.A.; Green, P.; Perry, T.L. Diet app use by sports dietitians: A survey in five countries. Jmir Mhealth Uhealth 2015, 3, e7. [CrossRef]

9. Pagoto, S.; Schneider, K.; Jojic, M.; DeBiasse, M.; Mann, D. Evidence-based strategies in weight-loss mobile apps. Am. J. Prev. Med. 2013, 45, 576-582. [CrossRef]

10. RunRepeat. Available online: https://runrepeat.com/state-of-running (accessed on 2 February 2021).

11. Morgan, D.W.; Martin, P.E.; Krahenbuhl, G.S. Factors affecting running economy. Sports Med 1989, 7, 310-330. [CrossRef]

12. Thomas, D.Q.; Fernhall, B.; GRANAT, H. Changes in running economy during a 5-km run in trained men and women runners. J. Strength Cond. Res. 1999, 13, 162-167.

13. Ribas-Barba, L.; Serra-Majem, L.; Román-Vinas, B.; Ngo, J.; García-Álvarez, A. Effects of dietary assessment methods on assessing risk of nutrient intake adequacy at the population level: From theory to practice. Br. J. Nutr. 2009, 101, S64-S72. [CrossRef]

14. Wei, J.; Vinnikova, A.; Lu, L.; Xu, J. Understanding and Predicting the Adoption of Fitness Mobile Apps: Evidence from China. Health Commun. 2020, 1-12. [CrossRef]

15. Wang, Z.; Derr, T.; Yin, D.; Tang, J. Understanding and predicting weight loss with mobile social networking data. In Proceedings of the 2017 ACM on Conference on Information and Knowledge Management, Singapore, 6-10 November 2017; pp. 1269-1278. [CrossRef]

16. Iacovides, S.; Meiring, R.M. The effect of a ketogenic diet versus a high-carbohydrate, low-fat diet on sleep, cognition, thyroid function, and cardiovascular health independent of weight loss: Study protocol for a randomized controlled trial. Trials 2018, 19, 62. [CrossRef]

17. Burke, L.M.; Sharma, A.P.; Heikura, I.A.; Forbes, S.F.; Holloway, M.; McKay, A.K.; Bone, J.L.; Leckey, J.J.; Welvaert, M.; Ross, M.L. Crisis of confidence averted: Impairment of exercise economy and performance in elite race walkers by ketogenic low carbohydrate, high fat (LCHF) diet is reproducible. PLoS ONE 2020, 15, e0234027. [CrossRef]

18. Weir, J.d.V. New methods for calculating metabolic rate with special reference to protein metabolism. J. Physiol. 1949, 109, 1-9. [CrossRef]

19. Saunders, P.U.; Pyne, D.B.; Telford, R.D.; Hawley, J.A. Reliability and Variability of Running Economy in Elite Distance Runners. Med. Sci. Sports Exerc. 2004, 36, 1972-1976. [CrossRef]

20. Achten, J.; Gleeson, M.; Jeukendrup, A.E. Determination of the exercise intensity that elicits maximal fat oxidation. Med. Sci. Sports Exerc. 2002, 34, 92-97. [CrossRef]

21. Bertuzzi, R.; Nascimento, E.M.; Urso, R.P.; Damasceno, M.; Lima-Silva, A.E. Energy system contributions during incremental exercise test. J. Sports Sci. Med. 2013, 12, 454.

22. Peronnet, F.; Massicotte, D. Table of nonprotein respiratory quotient: An update. Can. J. Sport Sci. 1991, 16, 23-29.

23. Amaro-Gahete, F.J.; Sanchez-Delgado, G.; Jurado-Fasoli, L.; De-la-O, A.; Castillo, M.J.; Helge, J.W.; Ruiz, J.R. Assessment of maximal fat oxidation during exercise: A systematic review. Scand. J. Med. Sci. Sports 2019, 29, 910-921. [CrossRef] [PubMed]

24. Martin, W.H.; Klein, S. Use of endogenous carbohydrate and fat as fuels during exercise. Proc. Nutr. Soc. 1998, 57, 49-54. [CrossRef] [PubMed]

25. Burke, L.M.; Angus, D.J.; Cox, G.R.; Cummings, N.K.; Febbraio, M.A.; Gawthorn, K.; Hawley, J.A.; Minehan, M.; Martin, D.T.; Hargreaves, M. Effect of fat adaptation and carbohydrate restoration on metabolism and performance during prolonged cycling. J. Appl. Physiol. 2000, 89, 2413-2421. [CrossRef] [PubMed]

26. Stellingwerff, T.; Spriet, L.L.; Watt, M.J.; Kimber, N.E.; Hargreaves, M.; Hawley, J.A.; Burke, L.M. Decreased PDH activation and glycogenolysis during exercise following fat adaptation with carbohydrate restoration. Am. J. Physiol. Endocrinol. Metab. 2006, 290, E380-E388. [CrossRef]

27. Burke, L.M.; Hawley, J.A.; Angus, D.J.; Cox, G.R.; Clark, S.A.; Cummings, N.K.; Desbrow, B.; Hargreaves, M. Adaptations to short-term high-fat diet persist during exercise despite high carbohydrate availability. Med. Sci. Sports Exerc. 2002, 34, 83-91. [CrossRef]

28. Carey, A.L.; Staudacher, H.M.; Cummings, N.K.; Stepto, N.K.; Nikolopoulos, V.; Burke, L.M.; Hawley, J.A. Effects of fat adaptation and carbohydrate restoration on prolonged endurance exercise. J. Appl. Physiol. 2001, 91, 115. [CrossRef] 
29. Burke, L.M.; Whitfield, J.; Heikura, I.A.; Ross, M.L.; Tee, N.; Forbes, S.F.; Hall, R.; McKay, A.K.; Wallett, A.M.; Sharma, A.P. Adaptation to a low carbohydrate high fat diet is rapid but impairs endurance exercise metabolism and performance despite enhanced glycogen availability. J. Physiol. 2020. [CrossRef]

30. Romijn, J.; Coyle, E.; Sidossis, L.; Gastaldelli, A.; Horowitz, J.; Endert, E.; Wolfe, R. Regulation of endogenous fat and carbohydrate metabolism in relation to exercise intensity and duration. Am. J. Physiol. Endocrinol. Metab. 1993, 265, E380-E391. [CrossRef]

31. Shaw, D.M.; Merien, F.; Braakhuis, A.; Maunder, E.; Dulson, D.K. Effect of a Ketogenic Diet on Submaximal Exercise Capacity and Efficiency in Runners. Med. Sci. Sports Exerc. 2019, 51, 2135-2146. [CrossRef]

32. Havemann, L.; West, S.J.; Goedecke, J.H.; Macdonald, I.A.; St Clair Gibson, A.; Noakes, T.; Lambert, E.V. Fat adaptation followed by carbohydrate loading compromises high-intensity sprint performance. J. Appl. Physiol. 2006, 100, 194-202. [CrossRef]

33. McSwiney, F.T.; Wardrop, B.; Hyde, P.N.; Lafountain, R.A.; Volek, J.S.; Doyle, L. Keto-adaptation enhances exercise performance and body composition responses to training in endurance athletes. Metabolism 2018, 81, 25-34. [CrossRef]

34. Bale, P.; Bradbury, D.; Colley, E. Anthropometric and training variables related to $10 \mathrm{~km}$ running performance. Br. J. Sports Med. 1986, 20, 170-173. [CrossRef]

35. Thomas, D.T.; Erdman, K.A.; Burke, L.M. Position of the Academy of Nutrition and Dietetics, Dietitians of Canada, and the American College of Sports Medicine: Nutrition and athletic performance. J. Acad. Nutr. Diet. 2016, 116, 501-528. [CrossRef]

36. Boushey, C.; Spoden, M.; Zhu, F.; Delp, E.; Kerr, D. New mobile methods for dietary assessment: Review of image-assisted and image-based dietary assessment methods. Proc. Nutr. Soc. 2017, 76, 283-294. [CrossRef]

37. Khanna, N.; Boushey, C.J.; Kerr, D.; Okos, M.; Ebert, D.S.; Delp, E.J. An overview of the technology assisted dietary assessment project at Purdue University. In Proceedings of the 2010 IEEE International Symposium on Multimedia, Taichung, Taiwan, 13-15 December 2010; pp. 290-295. [CrossRef]

38. Stepto, N.K.; Carey, A.L.; Staudacher, H.M.; Cummings, N.K.; Burke, L.M.; Hawley, J.A. Effect of short-term fat adaptation on high intensity training. Med. Sci. Sports Exerc. 2002, 34, 449-455. [CrossRef]

39. Jeukendrup, A.E.; McLaughlin, J. Carbohydrate ingestion during exercise: Effects on performance, training adaptations and trainability of the gut. In Sports Nutrition: More Than Just Calories-Triggers for Adaptation; Karger Publishers: Basel, Switzerland, 2011; Volume 69, pp. 1-18. [CrossRef]

40. Pfeiffer, B.; Stellingwerff, T.; Hodgson, A.B.; Randell, R.; Pöttgen, K.; Jeukendrup, A.E. Nutritional intake and gastrointestinal problems during competitive endurance events. Med. Sci. Sports Exerc. 2012, 44, 344-351. [CrossRef]

41. Simpson, A.; Gemming, L.; Baker, D.; Braakhuis, A. Do image-assisted mobile applications improve dietary habits, knowledge, and behaviours in elite athletes? A pilot study. Sports 2017, 5, 60. [CrossRef] 